\title{
Parieto-Frontal Circuits During Observation of Hidden and Visible Motor Acts in Children. A High-density EEG Source Imaging Study
}

\author{
Cristina Berchio - Tonia A. Rihs • Christoph M. Michel • \\ Denis Brunet · Fabio Apicella • Filippo Muratori • \\ Vittorio Gallese • Maria A. Umiltà
}

Received: 13 April 2013/Accepted: 27 August 2013/Published online: 12 September 2013

(C) Springer Science+Business Media New York 2013

\begin{abstract}
Several studies showed that in the human brain specific premotor and parietal areas are activated during the execution and observation of motor acts. The activation of this premotor-parietal network displaying the so-called Mirror Mechanism (MM) was proposed to underpin basic forms of action understanding. However, the functional properties of the MM in children are still largely unknown. In order to address this issue, we recorded high-density EEG from 12 children (6 female, 6 male; average age 10.5, $\mathrm{SD} \pm 2.15)$. Data were collected when children observed video clips showing hands grasping objects in two different experimental conditions: (1) Full Vision, in which the motor act was fully visible; (2) Hidden, in which the interaction between the hand and the object was not visible. Event-related potentials (ERPs) and topographic map analyses were used to investigate the temporal pattern of the ERPs and their brain source of localization, employing a children template of the Montreal Neurological Institute. Results showed that two different parieto-premotor circuits
\end{abstract}

C. Berchio $(\bowtie) \cdot$ V. Gallese · M. A. Umiltà

Section of Physiology, Department of Neuroscience, University

of Parma, Via Volturno 39/E, 43125 Parma, Italy

e-mail: cristina.berchio@unige.ch

Present Address:

C. Berchio - T. A. Rihs - C. M. Michel - D. Brunet

Functional Brain Mapping Laboratory, Department of

Fundamental Neurosciences, University of Geneva, 1211

Geneva, Switzerland

C. M. Michel

University Hospital, Geneva, Switzerland

F. Apicella · F. Muratori

Stella Maris Scientific Institute, University of Pisa, Via dei

Giacinti, 2, 56018 Calambrone, Pisa, Italy are activated by the observation of object-related hand reaching-to-grasping motor acts in children. The first circuit comprises the ventral premotor and the inferior parietal cortices. The second one comprises the dorsal premotor and superior parietal cortices. The activation of both circuits is differently lateralized and modulated in time, and influenced by the amount of visual information available about the hand grasping-related portion of the observed motor acts.

Keywords Brain circuits - Children - ERP · Mirror Mechanism · Source localization

\section{Introduction}

Human social life is based on the capacity to understand the intentions behind the behavior of other people. For several years, it has been hypothesized that action understanding may be founded on the capacity to read and represent mental states of other people (a theory of mind). The discovery of Mirror Neurons (di Pellegrino et al. 1992; Gallese et al. 1996; Rizzolatti et al. 1996) and the subsequent clarification of its functional properties in the premotor cortex (Kohler et al. 2002; Umiltà et al. 2001), has highlighted that action understanding may be based, at least at a basic level, on an embodied mechanism: the Mirror Mechanism (MM) (for a review see Gallese and Sinigaglia 2011).

Since the discovery of Mirror Neurons in macaque monkeys, several studies have described the existence of a MM in the human brain (for a review, see Rizzolatti and Sinigaglia 2010; Gallese and Sinigaglia 2011; Molenberghs et al. 2012). Few brain imaging and neurophysiological studies provide preliminary evidence of a possible role of 
the human MM in mapping basic motor intentions, like eating, drinking, putting objects away (Iacoboni et al. 2005; Cattaneo et al. 2007). Iacoboni et al. (2005) demonstrated that ventral premotor cortex responds differently to the observation of different motor intentions associated with grasping like drinking or cleaning up. Brass et al. (2007) showed activation of the MM when observing unusual actions like switching on the light with a knee, both when plausible (agent's hands are occupied) or not (agent's hands are free) to observers.

A recent EEG study carried out on healthy adults during action observation addressed the issue of the activation timing of the parieto-frontal MM during the observation of hand actions embedded in a context, suggesting the possible related motor intention (i.e. "grasping for eating"), or of hand actions without a context (Ortigue et al. 2010). Results showed that early left hemisphere activation was followed by right hemisphere activation. The authors of this study interpreted the "left" parieto-frontal activation as playing an important role in understanding the "goal" of the observed action (i.e. "grasping an object"), while the "right" parieto-frontal activation was thought to be involved in coding the motor intention of the observed action (i.e. "grasping an object for using it" or "grasping an object for placing it").

Despite several studies using different techniques reported evidence of a parieto-frontal MM in the adult human brain, several questions concerning its ontogenesis and development are still open (Lepage and Théoret 2007; Gallese et al. 2009, 2012; Marshall and Meltzoff 2011). Preliminary evidence from macaques suggests that a form of mirroring might be present at birth (Ferrari et al. 2012). However, no data are available on human neonates soon after birth. Furthermore, how the MM is shaped and modeled during development remains a still poorly charted territory.

In infants, EEG studies investigated the existence of shared brain patterns between action observation and execution through the study of a specific rhythm, the mu/ sensory-motor alpha rhythm, which is believed to be indirectly correlated to the MM in humans (for a review, see Pineda 2005). Several findings suggested that the suppression of 6-9 Hz EEG rhythm at central sites reflects the reactivity of infants' MM (Nyström 2008; Nyström et al. 2011; van Elk et al. 2008; Southgate and Csibra 2009; Reid et al. 2011).

Other empirical evidence documented the existence of a putative MM in school-age children. Lepage and Théoret (2006) measured mu suppression in children during hand grasping execution/observation. The authors found that mu rhythm suppression occurring during action execution was also present during observation of the same action. The EEG pattern described in this study resembles that shown in studies conducted on adults participants (Muthukumaraswamy and Johnson 2004; Muthukumaraswamy et al. 2004; Streltsova et al. 2010).

Marshall and Meltzoff (2011) underlined that most of the empirical evidence in children comes from studies that have investigated the $\mathrm{MM}$ in autism spectrum disorder (Oberman et al. 2005, 2008, 2012; Dapretto et al. 2006; Bernier et al. 2007; Cattaneo et al. 2007; Boria et al. 2009; Fabbri-Destro et al. 2009), and that the typical functioning of the MM was investigated for control purposes only. These studies documented the activity of the MM in children either during simple hand movements or during hand goal directed motor acts.

Few studies investigated the MM in children by means of fMRI, hence very little is known about the cortical localization of the MM at that age. One such study investigated the MM in ASD and typically developing (TD) children during observation/imitation of facial emotions (Dapretto et al. 2006). These authors reported that during the observation/imitation of facial emotional expressions TD children activated brain regions similar to those previously reported in adults: bilateral extra-striate visual cortices, premotor regions, limbic structures (amygdala, insula and ventral striatum) and the cerebellum. In particular, TD children showed strong bilateral activity in Brodmann's area 44 within the pars opercularis of the inferior frontal gyrus, as well as in Brodmann's area 45 (pars triangularis), with strongest peaks in the right hemisphere.

On the basis of this concise overview of the available data on action observation in school-age children, it appears that much is still to be known about the development, functionality and cortical localization of the action observation network. To this purpose, we employed highdensity electrical neuroimaging to explore and describe the cortical circuits and the temporal pattern of the activation of this network in a population of school-age TD children during the observation of hand grasping motor acts performed in two different conditions: (1) Full Vision condition $(\mathrm{FVc})$, in which the reaching-to-grasp motor act was fully visible; (2) Hidden condition ( $\mathrm{Hc}$ ), in which the handobject interaction was hidden by an interposed black screen.

\section{Materials and Methods}

\section{Subjects}

We recorded EEG signals from one group of 17 TD children. Children were recruited through the IRCCS Fondazione Stella Maris (Calambrone, Pisa, Italy). Five subjects were excluded due to excessive EEG artefacts. Thus, in the 
final sample 12 children were included: 6 female, 6 male (mean age 10.5, standard deviation \pm 2.15 ). All children had normal or corrected to normal visual acuity, no history of psychiatric or neurological impairments and were right handed.

The present study was approved by an appropriate local ethical committee and was performed in accordance with ethical standards. All parents gave their informed consent to the inclusion of the children in the study.

\section{Stimuli and Procedure}

The experiment consisted of three conditions (Fig. 1): (1) observation of video-clips of a hand grasping an object (FVc); (2) observation of video-clips of a hand grasping an object, in which the interaction between the hand and the object was occluded to sight (Hc); (3) observation of a black screen (Baseline Condition). To maintain the children's attention, several types of objects were employed as grasping targets: toys (a little cow, model of a car), 3D spheres (small and big), tools (mobile phone, a bunch of keys).

Experimental conditions were presented by means of E-Prime 2.0 (Psychology Software Tools). Movies were displayed at a viewing distance of $80 \mathrm{~cm}$. The videos were created at $30 \mathrm{frames} / \mathrm{s}$, size of $480 \times 720$ pixels. To minimize perceptive differences between conditions, the black screen of the Hc was added on the Full Vision videoclips by means of a specific software of video editing (Adobe Premiere Pro 2.0). Furthermore, in order to reduce eye movement artefacts, the movies were presented in the centre of a black background, resized to $50 \%$ both in width and in height of the original dimension.

Each trial started with a white fixation cross presented in the center of a black screen (randomized duration of 450-550 ms) (Fig. 1b). The fixation cross was followed by video clips: each movie lasted for $2 \mathrm{~s}$ and was repeated four times consecutively. Block were randomly presented within the same participant and across subjects. Overall, each of the three conditions was run 72 times. Furthermore, to ensure that participants paid attention to the videos, an attentive condition was included in the paradigm (Fig. 1b): at the end of each block a puppet appeared for $500 \mathrm{~ms}$ and children were instructed to say its name within a time window of $2 \mathrm{~s}$. The attentive condition was followed by the Baseline Condition (randomized in length from 2,500 to 3,500 ms).

For the duration of the experimental session the scene was video recorded by means of a video camera synchronized to the EEG acquisition system. The camera was placed in front of the children with the aim to monitor their behaviour.
Fig. 1 Stimuli and experimental paradigm. a Time line of the FVc and Hc. In the first $250 \mathrm{~ms}$, in both conditions, the object appears. From 250 to $500 \mathrm{~ms}$, in FVc the object is still visible, in the Hc a black screen appears and occludes the object. From 500 to $750 \mathrm{~ms}$, in both conditions, the hand appears approaching the object ("reaching phase"). From 750 to $1,000 \mathrm{~ms}$, in FVc the hand grasps the object, in the Hc the hand completely disappears behind the black screen ("grasping phase"). From 1,000 to $2,000 \mathrm{~ms}$, in the FVc the hand holds the object, in the $\mathrm{Hc}$ the hand remains behind the black screen. The dotted rectangle indicates the reaching and the grasping phase.

b Experimental sequence procedure: fixation cross, videoclips presentation, attentional task, baseline
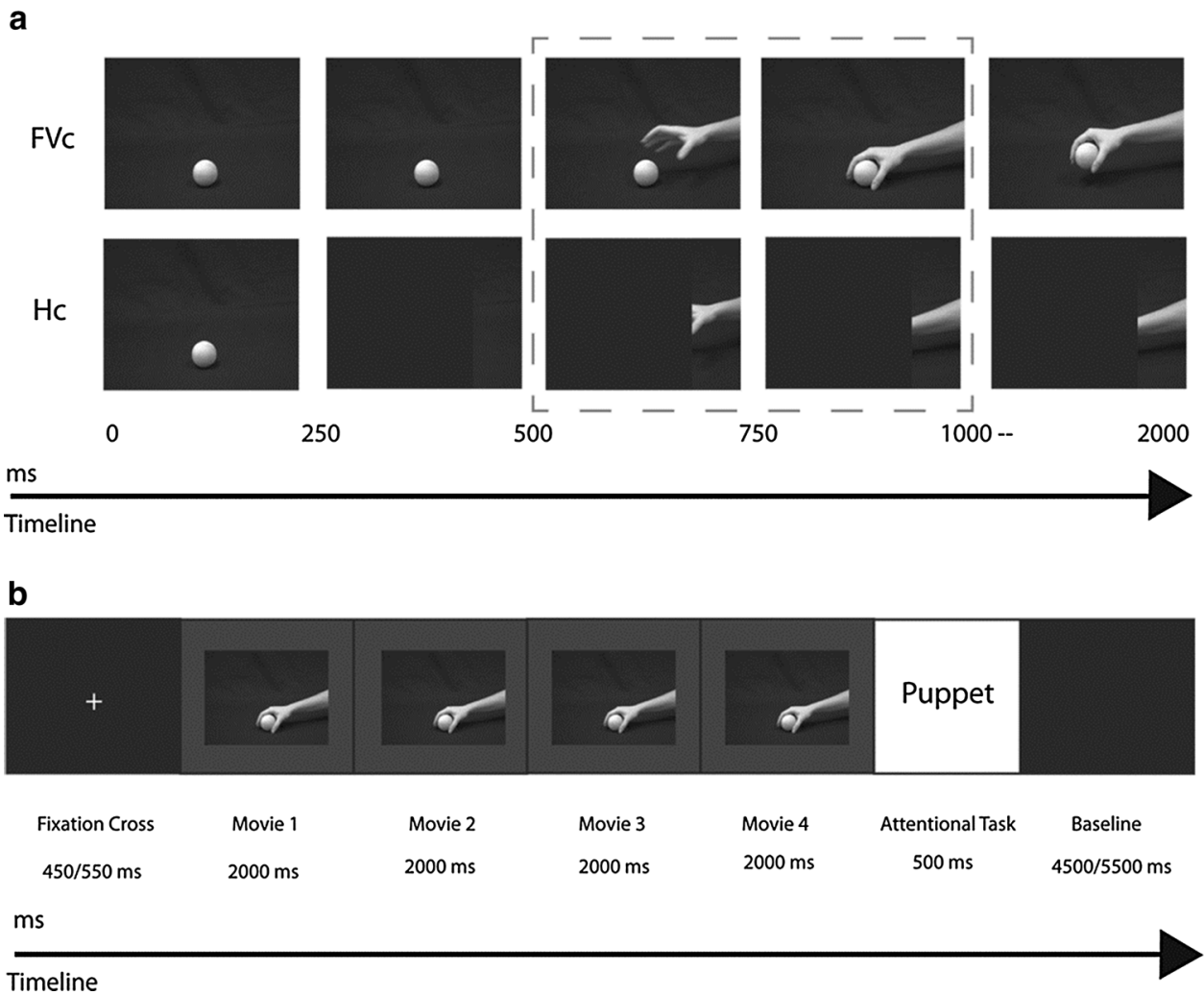
EEG Recordings and Pre-processing

The EEG was recorded by a net of 128 channels (Electrical Geodesic Inc., Eugene, OR, USA), at a sampling rate of $250 \mathrm{~Hz}$, filtered with a bandpass filter set to $0.3-100 \mathrm{~Hz}$ and using $\mathrm{Cz}$ as reference. For each electrode the impedance was kept below $50 \mathrm{k} \Omega$. Offline analysis was performed using the Cartool 3.52 software (http://brainmapping.unige.ch/ cartool.htm). EEG epochs were cut from $-100 \mathrm{~ms}$ (before stimulus onset) to $1,000 \mathrm{~ms}$ (after the begin of the stimulus) and separately averaged for the FVc and Hc. Although EEG epochs were cut from -100 to $1,000 \mathrm{~ms}$, since the main purpose of this experiment was to investigate the temporal dynamics of MM activations we focused from 500 to 1,000 ms (see Fig. 2): from the onset of the motor act to the achievement of its goal (the actual grasping of the object).

A bandpass filter from 1 to $30 \mathrm{~Hz}$ was applied off-line, and data were re-referenced to the common average reference. Bad channels were interpolated using the 3D spline interpolation method implemented in the Cartool software. Epoch averaging was performed after eliminating EEG artefacts. Channels with an amplitude exceeding $\pm 65 \mu \mathrm{V}$ were detected, and trials were excluded. An additional visual inspection was performed by two independent experimenters. Overall, a mean of 47 "clean" trials were retained in the FVc and a mean of 46 "clean" trials were retained in the Hc. Independent, two tailed $t$ tests conducted on the accepted number of trials for each child, showed no differences in trial rejections between conditions $(p=0.7)$. For subsequent analysis, peripheral channels located in the nape, were excluded and the original template was reduced from 128 to 110 channels.

\section{EEG Surface Analysis}

\section{Amplitude Analysis}

A widely employed approach for event-related potential (ERP) analysis, is to measure amplitude and latency of particular deflections in specific channels and fixed time windows. However, there are more exhaustive methods (Michel et al. 2004, p 120): "Instead of restricting the analysis to certain electrodes and a certain time window, the amplitude comparison can be extended to all electrodes and all time points". Instead of investigating pre-selected electrodes, this method allows for an investigation of amplitude differences on all channels and time-points. The advantage of this type of analysis are several: the experimental effects can be checked in all the electrodes and in all time points.

Differences in amplitude between the FVc and Hc were tested for each of the 110 electrodes and time points (from 500 to $1,000 \mathrm{~ms}$ ) by means of multiple $t$ tests. The
Fig. 2 Butterfly montage of the grand average for the $\mathrm{Hc}$ and the FVc. The GFP and the DISS index are also displayed. Dotted rectangle displays the time window of analysis. $H c$ hidden condition, $F V c$ full vision condition, GFP global field power, DISS dissimilarity

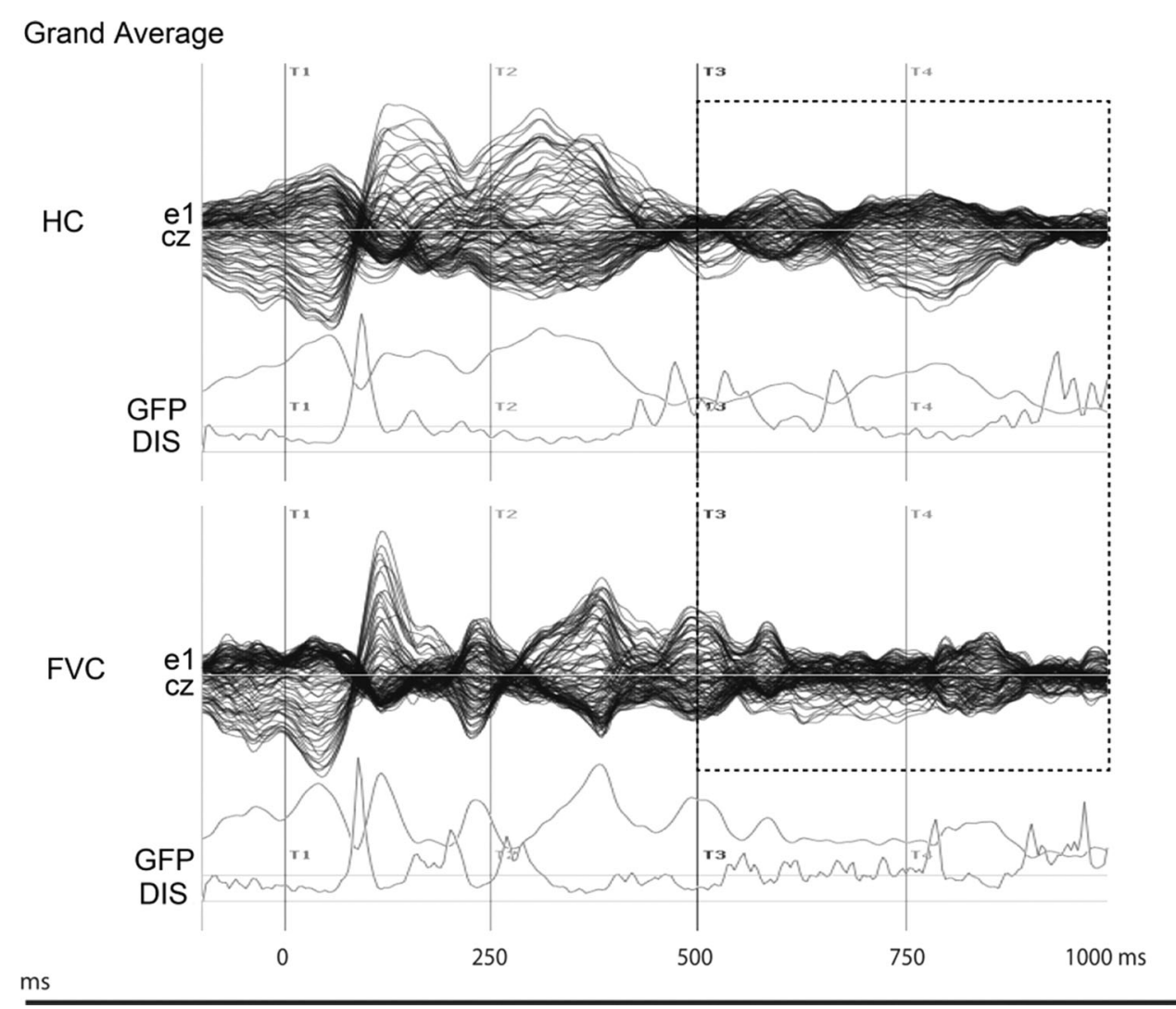


significance level was set to $p$ values $<0.01$, and a time constraint of $20 \mathrm{~ms}$ was applied (time constraint: activations were considered statistically significant only if lasting for $20 \mathrm{~ms}$ or longer).

With the aim of controlling for multiple comparisons, we performed an additional analysis: the randomization test (implemented in Cartool Software). The randomization test was done on the average across subjects of the variables tested (effects with $p$ values lower than 0.01 were considered significant only if lasting for $20 \mathrm{~ms}$ or longer).

\section{Analysis of Field Topography: TANOVA}

The EEG scalp maps represent the voltage potential field on the whole head and are characterized by particular "landscapes" (topography). There are several explanations for basing analyses on topography maps (Murray et al. 2008; Michel and Murray 2012). The "traditional" approach of ERPs focuses on waveform morphology: at fixed time window, at specific electrode positions. However, the waveforms analysis neglects important properties of multichannel EEG: the spatial characteristics of the electric fields at the scalp and the temporal dynamics of these fields. Indeed, the voltage potential field on the whole scalp is characterized by a specific topography and this field topography is directly related to the underlying brain sources. Consequently, analyzing the whole electric field topography, and looking for topographical differences between experimental conditions allows to detect temporal instants when different neuronal populations were active in the brain. Additionally, as compared to "traditional waveforms approach", the analysis of the topography of the electric fields has another important advantage: it is completely reference independent (see Michel et al. 2004; Murray et al. 2008).

Two measures are commonly used to describe high-density EEG topographies: the global field power (GFP) and the global map dissimilarity (DISS) (see Fig. 2). The GFP is the standard deviation of the average referenced potentials over all electrodes and is a measure of map strength. Periods of high GFP correspond to periods of stable map configurations. To indicate periods of map transitions, the DISS is used, which is a difference measure between two adjacent maps, independent of their map strength (i.e. the root mean square of the difference between two maps normalized by the GFP) (Michel and Murray 2012). It is thus a global measure of topographic differences between scalp maps. Despite the name, the TANOVA is a non-parametric randomization test that compares the DISS between conditions (for more detailed explanations see Michel et al. 2004; Michel and Murray 2012).

This is done in the following way (Brunet et al. 2011): first, assigning the maps of the single participant in a randomized way to each experimental conditions; second, re-measuring the group-average ERPs; third, re-measure the resulting GMD value for these "new" group-average ERPs.

In order to test for differences in topography between the $\mathrm{FVc}$ and the $\mathrm{Hc}$, in terms of their field configurations, a "topographic ANOVA" (TANOVA) was implemented. The TANOVA was performed in our windows of investigation (500-1,000 ms) and effects with $p$ values lower than 0.05 were considered significant only if lasting for $20 \mathrm{~ms}$ or longer (time constraint: consecutive durations $>20 \mathrm{~ms}$ ).

\section{Analysis of Stimulus-Evoked Sources}

In order to measure the stimulus evoked sources we applied a Local Auto Regressive Average model (LAURA, Grave de Peralta Menendez et al. 2001). LAURA belongs to the family of distributed linear inverse solution (Michel et al. 2004).

We calculated the average intracranial source distribution for each condition, for all children. An anatomically constrained head model (L-SMAC model, Brunet et al. 2011; Spinelli et al. 2000) was used. The solution space consisted in 3,007 solution points within the grey matter of a MNI pediatric average brain, ranging in age from 7.5 to 13.5 years (NIHPD324 pediatric template; Fonov et al. 2009, 2011).

By means of a nonlinear transformation the brain coordinates were converted from MNI into Talairach space (Brett 2006, http://imaging.mrc-cbu.cam.ac.uk/imaging/ MniTalairach).

To compare the source distribution between the two conditions we performed two separate steps of analysis. First, to evaluate the conditions separately with regard to the Baseline Condition, statistical comparisons were conducted by performing paired $t$ tests at each solution point ("voxel-by-voxel" paired $t$ test), between each condition and the Baseline Condition. This first step of analysis was performed in order to obtain a contrast between MM activation and rest. We then compared the $\mathrm{FVc}$ and $\mathrm{Hc}$ directly (Contrast Analysis). A voxel-by-voxel paired $t$ test was applied for the Contrast Analysis.

All Stimulus Evoked Source analyses were computed in two time windows: from 500 to $760 \mathrm{~ms}$; from 760 to $1,000 \mathrm{~ms}$. Only significant differences with $p$ values $<0.05$ and consecutive durations of $20 \mathrm{~ms}$ are reported here (time constraint: consecutive durations $>20 \mathrm{~ms}$ ).

In order to evaluate the reliability of the multiple comparisons between each condition and the Baseline Condition and the reliability of the multiple comparisons between the 'Contrast Analysis' a randomization test was performed (effects with $p$ values lower than 0.05 were considered significant only if lasting for $20 \mathrm{~ms}$ or longer). 


\section{Results}

\section{EEG Surface Analysis}

\section{Amplitude Analysis}

To investigate differences between conditions, we compared the amplitude differences between the FVc and the $\mathrm{Hc}$ from the beginning of the reaching to the end of grasping (from 500 to $1,000 \mathrm{~ms}$ ) at each electrode (see Fig. 3a).

The amplitude analysis showed significant differences in five time windows (see Fig. 3b). At the beginning of "reaching" (500-530 ms) a stronger positivity was observed in the FVc on left occipital electrodes (around $01 ; p=0.002, t=+4.5$ ), with a maximum peak at $510 \mathrm{~ms}$. Afterwards, from 550 to $670 \mathrm{~ms}$, more negative amplitudes were recorded in the $\mathrm{Hc}$ at right parietal and central sites. More precisely, around P8 with a maximum at $600 \mathrm{~ms}(p=0.001, t=-2.4)$ and around $\mathrm{C} 4$ and P4 with a maximum at $625 \mathrm{~ms}(p=0.001, t=-4)$. At the end of the "reaching phase", in the time window from 690 to
$810 \mathrm{~ms}$, the most significant differences were found at parietal right electrodes P3 and P7 (maximum at $780 \mathrm{~ms}$; $p=0.001, t=+1.4$ ) with more positive potentials in the FVc. During the "grasping phase", from 830 to $900 \mathrm{~ms}$, the evoked potentials showed a more negative deflection in central-parietal sites for the $\mathrm{Hc}$, in correspondence to $\mathrm{C} 3$ and P3 electrodes with a maximum at $865 \mathrm{~ms}(p=0.002$, $t=-2.2$ ). At the end of the "grasping phase" (920$970 \mathrm{~ms}$ ), ERPs became more positive in FVc at central electrodes (around C4 at $990 \mathrm{~ms} ; p=0.003, t=+2$ ).

All the significant differences described above (see Fig. 3b) were confirmed by the randomization test ( $p \mathrm{~s}<0.001 ;>20 \mathrm{~ms})$.

\section{TANOVA}

To investigate whether these amplitude differences were due to topographic modulations, we calculated the topographic analysis of variance between the two conditions for each time point. The TANOVA analysis showed that maps differ between conditions in five time windows: $500-540 \mathrm{~ms}$ $(p=0.004) ; \quad 575-645 \mathrm{~ms} \quad(p=0.002) ; \quad 720-765 \mathrm{~ms}$
Fig. 3 a Video clips of FVc and Hc. Single frames from 500 to $1,000 \mathrm{~ms}$ are shown. b Amplitude comparison between FVc and Hc. Channels are reported on the vertical axis, time is indicated on the horizontal axis. Black lines indicate significant $p$ values $(p<0.05)$. Dotted rectangles display the time windows of statistically significant differences between conditions. c TANOVA results. At the top, statistically significant periods of map differences are indicated by black bars $(p<0.05)$. Voltage maps that describe periods of differences are shown. Dotted rectangles indicate the time windows of statistically significant differences between conditions a

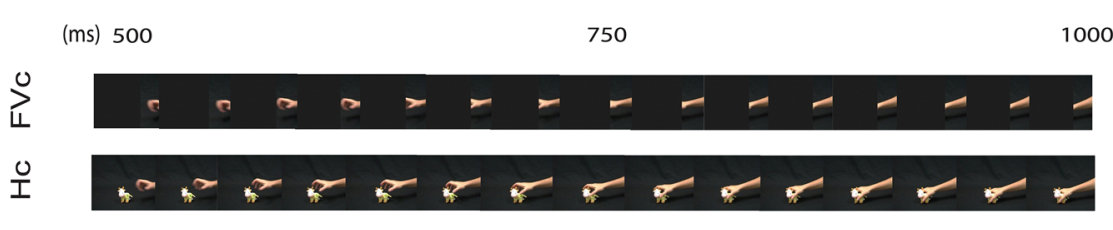

\section{b}
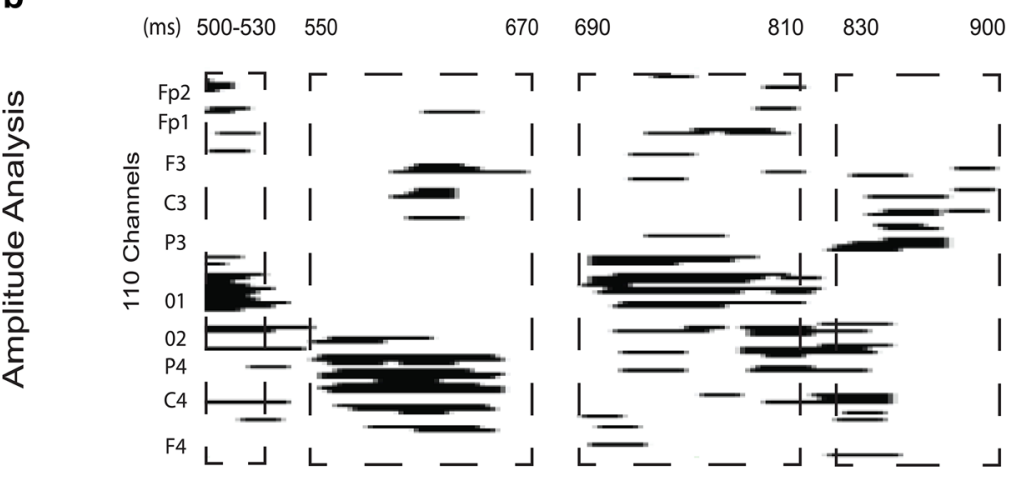

980-1000

Г

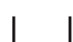

I
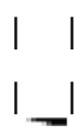

I I

ᄂ

C

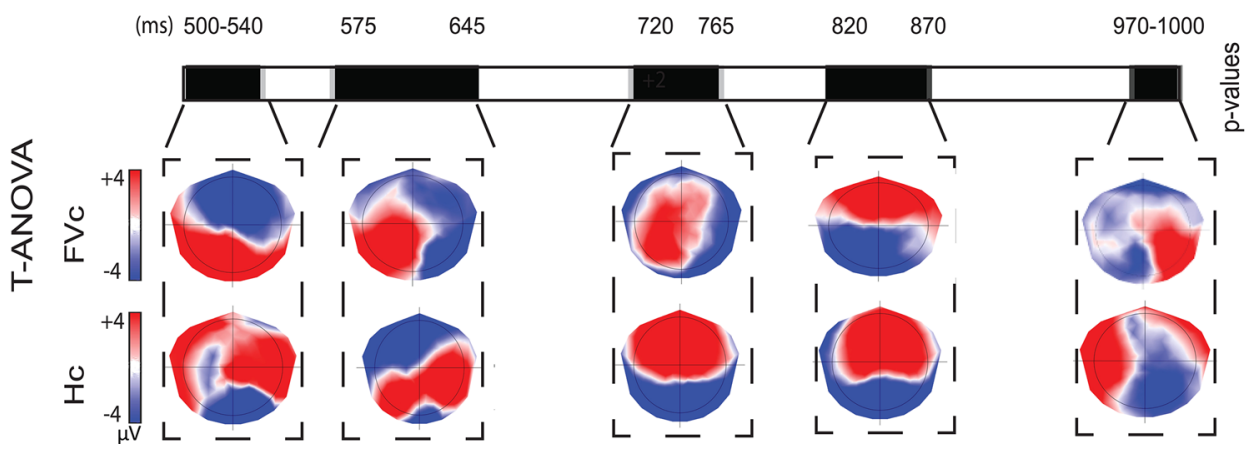


$(p=0.005) ; \quad 820-870 \mathrm{~ms} \quad(p=0.005) ; \quad 970-1,000 \mathrm{~ms}$ $(p=0.004)$. The TANOVA results are shown in Fig. 3c.

In summary, this topographic profile analysis highlighted several points. First, the manifestation of significant topographic differences between conditions becomes evident from the very beginning of the "reaching phase". Second, that the different temporal patterns shown by the waveform analysis, were widely overlapped with the different topographic modulations revealed by the TANOVA.

\section{Analysis of Stimulus-Evoked Sources}

We analyzed the time course of statistical differences in current source distribution in first two different steps: (a) between FVc and Baseline Condition and (b) between Hc and Baseline Condition. This approach allowed us to define the brain areas activated with respect to the Baseline. Furthermore, the sources activities were described with respect to the time windows detected by the TANOVA analysis.
For FVc (see Table 1), consistent activations (highlighted by positive $t$ values) were found: in frontal areas including the middle frontal gyrus (ventral left: $p=0.02$, $t=+2.6$; ventral right: $p=0.04, t=+2.4$; dorsal right: $p=0.01, t=+4.6)$, the superior frontal gyrus $(p=0.02$, $t=+2.7)$, in the inferior parietal lobe $(p=0.02$, $t=+2.8$ ) and in the superior parietal areas (superior parietal lobule $p=0.02, t=+2.8$; precuneus left: $p=0.04, t=+2.5$; precuneus right: $p=0.04, t=+2.1$ ). Additionally, left prefrontal activation $(p=0.01, t=+3)$ and occipital activations $(p=0.04, t=+2)$ were found. Table 1 summarizes the MNI and the Talairach coordinates, of the current source density maximum, for each of these foci.

For Hc (see Table 2), LAURA distributed inverse solution revealed frontal activations (highlighted by positive $t$ values): in the left ventral frontal gyrus $(p=0.01$, $t=+4.4)$, in the right dorsal frontal gyrus $(p=0.02$, $t=+3)$, in the left superior frontal gyrus $(p=0.04$, $t=+2.3)$. Several parietal activations were also detected:

Table 1 FVc: MNI and Talairach coordinates of the current source density maximum for each activation

\begin{tabular}{|c|c|c|c|c|c|c|c|c|}
\hline & \multicolumn{3}{|l|}{ MNI } & \multicolumn{3}{|c|}{ Talairach } & \multirow[b]{2}{*}{$t$} & \multirow[b]{2}{*}{$p$} \\
\hline & $x$ & $y$ & $z$ & $x$ & $y$ & $z$ & & \\
\hline \multicolumn{9}{|c|}{ Brodmann Area 6} \\
\hline \multicolumn{9}{|c|}{ Middle frontal gyrus (Ventral) } \\
\hline Left & -43 & 11 & 58 & -42 & 13 & 53 & 2.6 & 0.02 \\
\hline Right & 51 & 12 & 51 & 50 & 14 & 46 & 2.4 & 0.04 \\
\hline \multicolumn{9}{|c|}{ Middle frontal gyrus (Dorsal) } \\
\hline Right & 35 & 11 & 67 & 35 & 14 & 61 & 4.6 & 0.01 \\
\hline \multicolumn{9}{|c|}{ Superior frontal gyrus } \\
\hline Left & -27 & 27 & 58 & -19 & 27 & 67 & 2.7 & 0.02 \\
\hline Right & 27 & 4 & 75 & 24 & -1 & 70 & & 0.02 \\
\hline \multicolumn{9}{|c|}{ Brodmann Area 40} \\
\hline \multicolumn{9}{|c|}{ Inferior parietal lobule } \\
\hline Left & -43 & 11 & 58 & -43 & 13 & 53 & 2.8 & 0.02 \\
\hline \multicolumn{9}{|c|}{ Brodmann Area 7} \\
\hline \multicolumn{9}{|c|}{ Superior parietal lobule } \\
\hline Left & -32 & -54 & 60 & -31 & -49 & 58 & 2.8 & 0.02 \\
\hline \multicolumn{9}{|c|}{ Precuneus } \\
\hline Left & -7 & -55 & 60 & -7 & -50 & 57 & 2.5 & 0.04 \\
\hline Right & 6 & -54 & 60 & 6 & -50 & 57 & 2.1 & 0.04 \\
\hline \multicolumn{9}{|c|}{ Brodmann Area 8} \\
\hline \multicolumn{9}{|c|}{ Superior frontal gyrus } \\
\hline Left & -19 & 51 & 51 & -19 & 51 & 44 & 3 & 0.01 \\
\hline \multicolumn{9}{|c|}{ Brodmann Area 18} \\
\hline \multicolumn{9}{|c|}{ Lingual gyrus } \\
\hline Left & -11 & -74 & 4 & -11 & -71 & 7 & 2 & 0.04 \\
\hline Right & 11 & -74 & 4 & 11 & -71 & 7 & 2 & 0.04 \\
\hline
\end{tabular}


in the inferior parietal lobe bilaterally (left: $p=0.04$, $t=+2.4$; right: $p=0.01, t=+4)$, in the left superior parietal lobe $(p=0.04, t=+2.4)$, in the precuneus (left: $p=0.04, t=+2.2$; right: $p=0.03, t=+2.6)$ and in the post central gyrus $(p=0.02, t=+2.8)$. Furthermore, several occipital activations were found (cuneus left: $p=0.04, t=+2.3$; cuneus right: $p=0.02, t=+2.2$; lingual gyrus left: $p=0.04, t=+2.2$; lingual gyrus right:

Table 2 Hc: MNI and Talairach coordinates of the current source density maximum for each activation

\begin{tabular}{|c|c|c|c|c|c|c|c|c|}
\hline & \multicolumn{3}{|l|}{ MNI } & \multicolumn{3}{|c|}{ Talairach } & \multirow[b]{2}{*}{$t$} & \multirow[b]{2}{*}{$p$} \\
\hline & $x$ & $y$ & $z$ & $x$ & $y$ & $z$ & & \\
\hline \multicolumn{9}{|c|}{ Brodmann Area 6} \\
\hline \multicolumn{9}{|c|}{ Middle frontal gyrus (Ventral) } \\
\hline Left & -43 & 11 & 58 & -42 & 13 & 53 & 4.4 & 0.01 \\
\hline \multicolumn{9}{|c|}{ Middle frontal gyrus (Dorsal) } \\
\hline Right & 35 & 11 & 67 & 35 & 14 & 61 & 3 & 0.02 \\
\hline \multicolumn{9}{|c|}{ Superior frontal gyrus } \\
\hline Left & -19 & 27 & 67 & -19 & 29 & 60 & 2.3 & 0.04 \\
\hline Right & 5 & 11 & 67 & 5 & 14 & 61 & & \\
\hline \multicolumn{9}{|c|}{ Brodmann Area 40} \\
\hline \multicolumn{9}{|c|}{ Inferior parietal lobule } \\
\hline Left & -51 & -35 & 58 & -50 & -31 & 55 & 2.4 & 0.04 \\
\hline Right & 58 & -43 & 51 & 50 & -40 & 49 & 4 & 0.01 \\
\hline \multicolumn{9}{|c|}{ Brodmann Area 7} \\
\hline \multicolumn{9}{|c|}{ Superior parietal lobule } \\
\hline Left & -32 & -48 & 56 & -36 & -56 & 54 & 2.4 & 0.04 \\
\hline \multicolumn{9}{|c|}{ Precuneus } \\
\hline Left & -11 & -51 & 67 & -16 & -53 & 54 & 2.2 & 0.04 \\
\hline Right & 31 & -50 & 55 & 30 & -45 & 52 & 2.6 & 0.03 \\
\hline \multicolumn{9}{|c|}{ Postcentral gyrus } \\
\hline Left & -24 & -53 & 71 & -24 & -48 & 68 & 2.8 & 0.02 \\
\hline \multicolumn{9}{|c|}{ Brodmann Area 5} \\
\hline \multicolumn{9}{|c|}{ Postcentral gyrus } \\
\hline Left & -33 & -50 & 63 & -32 & -45 & 60 & 2.6 & 0.03 \\
\hline \multicolumn{9}{|c|}{ Brodmann Area 9} \\
\hline \multicolumn{9}{|c|}{ Medial frontal gyrus } \\
\hline Left & -4 & 54 & 43 & -4 & 54 & 37 & 2.2 & 0.04 \\
\hline Right & 4 & 54 & 43 & 4 & 54 & -37 & 2.5 & 0.03 \\
\hline \multicolumn{9}{|c|}{ Brodmann Area 10} \\
\hline \multicolumn{9}{|c|}{ Superior frontal gyrus } \\
\hline Right & 11 & 72 & 19 & 11 & 71 & 14 & 2.2 & 0.04 \\
\hline \multicolumn{9}{|c|}{ Brodmann Area 17} \\
\hline \multicolumn{9}{|l|}{ Cuneus } \\
\hline Left & -4 & -82 & 4 & -4 & 80 & 7 & 2.3 & 0.04 \\
\hline Right & 11 & -82 & 4 & 11 & -79 & 8 & 2.8 & 0.02 \\
\hline \multicolumn{9}{|c|}{ Brodmann Area 18} \\
\hline Lingual & & & & & & & & \\
\hline Left & -11 & -74 & 4 & -11 & -71 & 7 & 2.2 & 0.04 \\
\hline Right & 27 & -79 & 1 & 27 & -79 & 1 & 2.2 & 0.04 \\
\hline Brodmann & & & & & & & & \\
\hline Cuneus & & & & & & & & \\
\hline Left & -34 & -75 & 27 & -34 & -72 & 28 & 3.2 & 0.01 \\
\hline Right & 11 & -82 & 27 & 11 & -78 & 29 & 2 & 0.04 \\
\hline
\end{tabular}


$p=0.04, t=+2.2)$. Finally, prefrontal activations were found in the superior frontal gyrus $(p=0.04, t=2.2)$. For $\mathrm{Hc}$, the MNI and Talairach coordinates, of the current source density maximum, for each of these foci are display in Table 2.

To statistically validate whether these brain activations were different or not between conditions, we contrasted $\mathrm{FVc}$ and $\mathrm{Hc}$ (exemplificative activations are shown in Fig. 4).

The results of the Contrast Analysis are reported in Table 3. In the first time window detected by the TANOVA (500-540 ms), differences were found: in the premotor cortex (left middle frontal gyrus: $p=0.0$, $t=+2.9$; right superior frontal gyrus: $p=0.0, t=+3.2)$, in the left superior parietal lobule $(p=0.0, t=+2.32)$, in the inferior parietal lobule $(p=0.0, t=+3)$ with an increase of activation for $\mathrm{FVc}$ (highlighted by positive $t$ values); in visual areas ( $p=0.01, t=-4.9)$, and right prefrontal cortex ( $p=0.01, t=-4.4)$ with an increase of activations for $\mathrm{Hc}$ (highlighted by negative $t$ values). In the second time window identified by the TANOVA $(575-645 \mathrm{~ms})$ an increase of activation for $\mathrm{Hc}$ was found in the premotor cortex [in a left ventral part $(p=0.00$ $t=-3.2)$ and in a dorsal right part $(p=0.00 t=-2.2)]$, in the superior parietal lobule $(p=0.0, t=-2)$ and in occipital areas $(p=0.00 t=-3.2)$; an increase of activation for $\mathrm{FVc}$ was found in the superior frontal gyrus $(p=0.00, t=+2)$. In the third window detected by the TANOVA (720-765 ms) an increase of activation was found in the right ventral premotor cortex $(p=0.01$, $t=+2.5)$ for $\mathrm{FVc}$. In $\mathrm{Hc}$ increased activations were detected in the right inferior parietal lobe $(p=0.00$, $t=-4.38)$, in the left superior lobe $(p=0.00, t=$ $-2.45)$ and in other visual areas $(p=0.00, t=-4)$. In the fourth TANOVA window $(820-870 \mathrm{~ms})$, an increase of activation for Hc was found in the left dorsal premotor

Fig. 4 Electrical source imaging (LAURA) differences between FVc and Hc. Source estimations are rendered on the NIHPD324 pediatric template brain. Statistically significant activations during third TANOVA window are shown: 720-765 ms. a Red color corresponds to significant $p$ values. b $T$ values are shown. Orange-red colors indicate stronger current densities in $\mathrm{FVc}$ while blue-violet colors indicate stronger current densities in $\mathrm{Hc}$
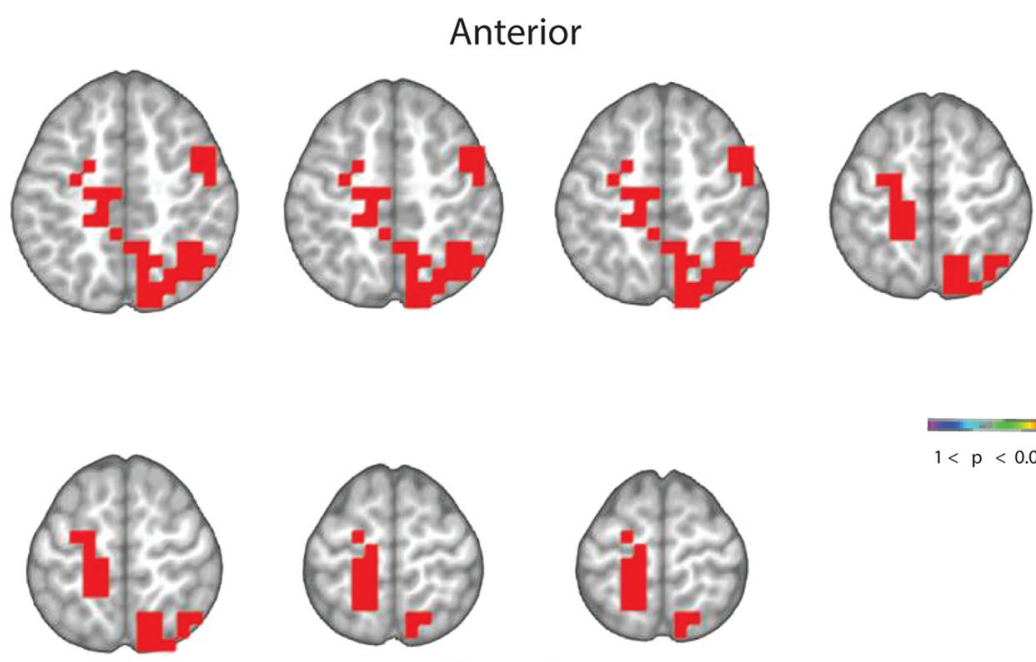

$1<\mathrm{p}<0.05$

Posterior

Anterior
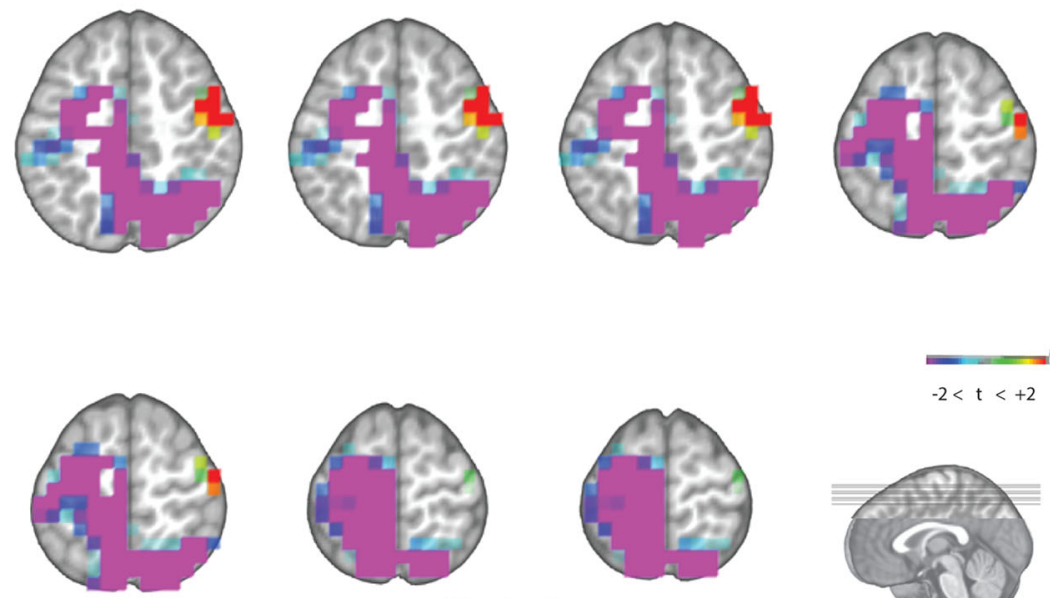

\section{Posterior}

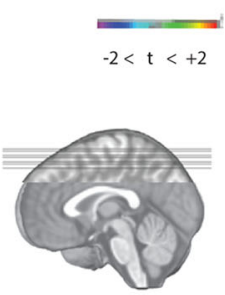

Full Vision Condition - Hidden Condition 
Table 3 Source localization of topographic maps: comparison between contrast analysis and baseline analysis

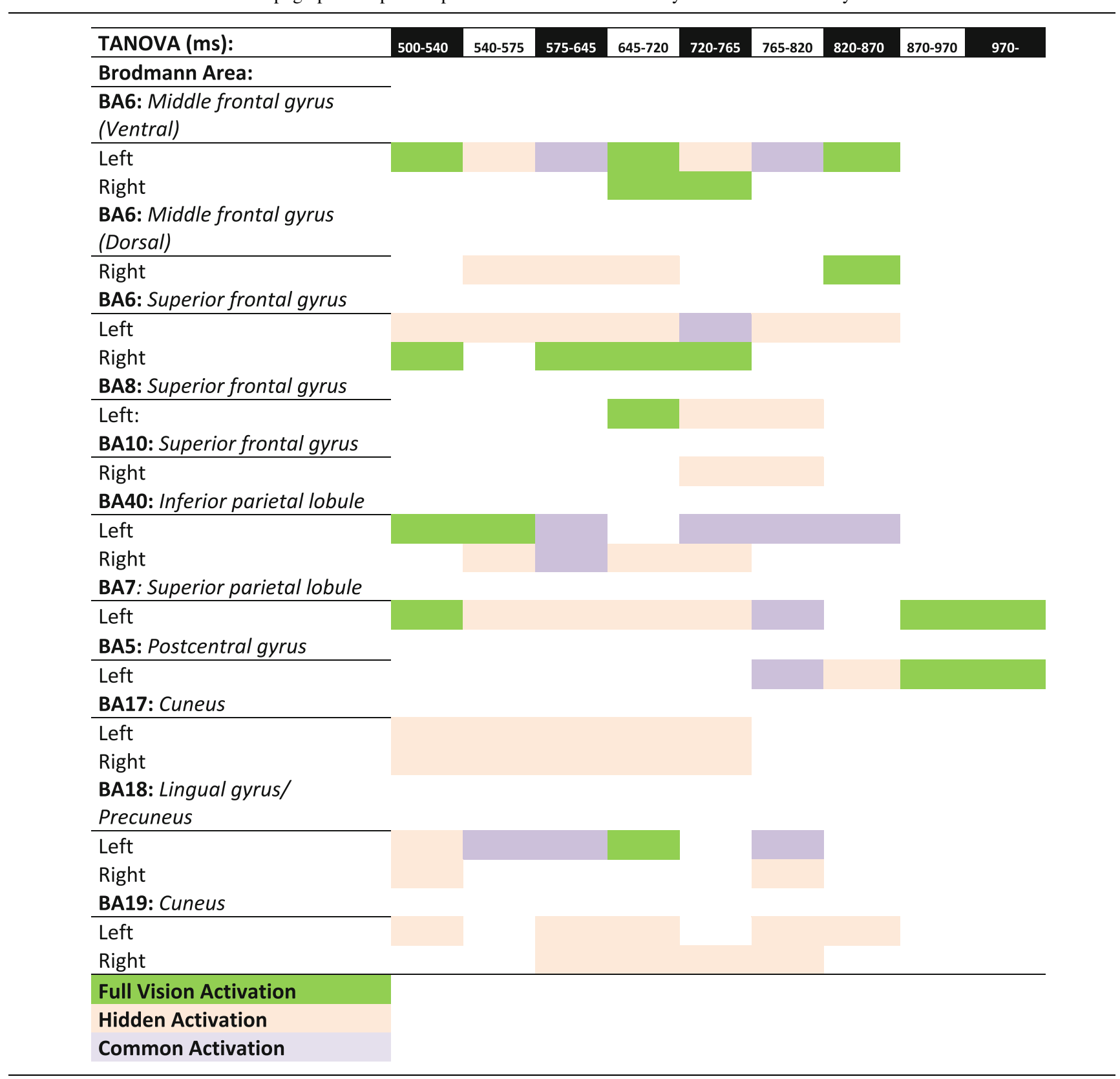

cortex $(p=0.00 t=-2.45)$ and in the left superior parietal lobe $(p=0.00, t=-1.7)$; for FVc: in the left middle frontal gyrus $(p=0.00, t=+2)$ and in the right dorsal premotor cortex $(p=0.00, t=+2)$. In the fifth TANOVA window (970-1,000 ms), an increase of activation for $\mathrm{FVc}$ was found in the superior parietal lobe $(p=0.00, t=+3)$.

It should be noted that some of the differences detected concerned overlapped activations (revealed by the previously analysis with Baseline Condition) and that these differences highlighted the intensity of shared processes involved. The Contrast Analysis, about these shared activations, revealed a stronger response in $\mathrm{Hc}$ than in $\mathrm{FVc}$ in the superior parietal lobule $(p=0.00, t=-2.45)$ (map: $765-820 \mathrm{~ms}$ ) and in the ventral middle frontal gyrus ( $p=0.00, t=-3.2$ ) (map: 575-645 ms).

The absence of differences in overlapped activations showed by the contrast between Hc and FVc was do to the fact that intensity of these responses were similar in both experimental conditions. 
All the significant differences reported in the inverse space were confirmed by the randomization test ( $p \mathrm{~s}<0.005 ;>20 \mathrm{~ms})$.

\section{Discussion}

Empirical evidence has shown that a mechanism whose functional properties are similar to the MM described in adults can be detected in early infancy (see Marshall and Meltzoff 2011). EEG studies conducted in infants and children, were mainly focused on the analysis of $\mathrm{Mu}$ rhythm (Nyström 2008; Nyström et al. 2011; van Elk et al. 2008; Southgate and Csibra 2009; Reid et al. 2011; Lepage and Théoret 2006). However, the use of more sophisticated techniques of investigation can shed new light on unsolved questions. In this respect, the estimation of the generators of the scalp recordings furthers our understanding of the action observation network. Although EEG source imaging methods are bound to mathematical constraints, recent progresses in spatial sampling density and signal analysis have rendered EEG a brain imaging method that is able to provide reliable spatial and temporal information (see Michel and Murray 2012).

By means of EEG source imaging method, in the present work we showed that in children the observation of fully visible and partially hidden hand grasping actions are both able to activate cortical areas traditionally thought to be part of the "grasping mirror circuit": the premotor cortex and the inferior parietal lobe (for a review, see Rizzolatti and Sinigaglia 2010; Molenberghs et al. 2012). In the present study we only focused on action observation. However, it must be taken into account that the activation of the motor system during action observation is considered as evidence of activation of the MM (see Rizzolatti and Sinigaglia 2010). In adults it is well known that the parietal cortex is involved in processing action-related information like: object shape, orientation, motion, knowledge about tools and action's understanding (Culham and Valyear 2006).

A recent EEG study by Ortigue et al. (2010) documented the time course of cortical activation during the observation of hand motor acts (object grasping and object touching) occurring with or without context (objects surrounding the target object, suggesting two different potential motor intentions, like grasping to drink from the object vs. grasping to move the object). At the beginning, diffuse posterior bilateral cortical activations were found for all investigated conditions. In a second phase, a more marked left activation appeared in the left posterior temporal cortices and in the inferior parietal cortices. Subsequently, increase of activations in right temporal and parietal regions together with bilateral frontal activations were detected. During this third phase, some differences were identified depending on the intentional transparency of the observed motor acts: more prolonged activations were found for more complex hand object interactions (i.e. grasping the object for transporting it vs. touching the object). In the last phase, a general decrease of activation was described.

As described in the "Results" section of our paper, FVc and Hc have shown different patterns of ERP waveform (showed by the TANOVA and the ERPs amplitude analysis), which are reflected in different timing of brain activation and processing. In FVc, during the early phase, coinciding with reaching and hand shaping, activation of the left ventral premotor cortex and of the left inferior parietal cortex were observed. During a later phase, coinciding with grasping completion, activation in the right ventral premotor cortex and of the right inferior parietal cortex was observed, while the previous left side activations disappeared. Although our results are similar to those of Ortigue et al. (2010), one crucial remark is required. In addition to parietal activation, our results show ventral premotor activation, thus highlighting that the observation of fully visible grasping triggers the entire "grasping mirror circuit" bilaterally. Interestingly, such bilateral response does not occur simultaneously. The left hemisphere is involved only during the first phase of grasping observation, while the right one prevails during a later phase. This seems to suggest possible functional differences of the right and left "grasping mirror circuit", which the poor time resolution of fMRI technique so far was not able to reveal.

Ortigue et al. (2010) suggested that during the observation of object-related hand actions two main processes take place to understand the agent's motor intention. First, motor act recognition would take place, in relation to the object semantics ("what the agent is doing?" i.e. grasping a cup); second, the understanding of the motor intention behind the observed motor act would ensue ("why the cup is grasped in that particular manner?" i.e. grasping the cup for drinking). According to these authors, the left inferior parietal lobe, because of its properties, could enable the observer to recognize what another person is doing, while the right inferior parietal activation could reflect the involvement of this area in motor intention understanding. These two processes are thought to be connected: if the first step is not accomplished, the second one cannot take place.

If we try to interpret our data according to this theoretical framework, it's possible to speculate that the left lateralized early activations we detected can underpin the "what" encoding of the MM. In other words, it is likely that when a motor act is "transparent", the MM encodes the observed motor act as "grasping" also in children. Our later right hemisphere parieto-premotor activation could in 
principle underpin the detection of the observed motor act's motor intention. However, given the lack of different contexts in our experimental paradigm, this remains a mere speculation. An alternative hypothesis could be that the right cortical activation might reflect the way in which the observed grasping motor act is performed ("how" component)

In $\mathrm{Hc}$, similarly to $\mathrm{FVc}$, during the early phase coinciding with reaching and hand shaping, activation of the left ventral premotor cortex and of the left inferior parietal cortex was observed. However, an additional response of the right inferior parietal lobe was detected. During the later phase, activation continued in the left ventral premotor cortex and in the left inferior parietal cortex, together with the previous right side parietal activation. Thus, in $\mathrm{Hc}$, activation mainly differs from that recorded in $\mathrm{FVc}$ in two aspects: first, the two phases of grasping observation basically produce the same pattern of cortical activity. Second, activation of right ventral premotor cortex never occurs. It is possible that the lack of visual information about how grasping is accomplished contributes to the disappearance of the right premotor activation, given that the "how" component of the observed motor act is not available to observers.

Another interesting result of the present study is that in both conditions the activated action observation network is not confined to the ventral premotor cortex and the inferior parietal lobe. Indeed, activations were detected also within the dorsal part of the premotor cortex and in the superior parietal lobule. These cortical locations are part of the "reaching circuit" (the transport phase of the hand towards a particular position in space), showing overlapping activation during reaching execution, observation and imagery (Filimon et al. 2007).

In adults, a recent analysis of the parieto-premotor mirror network (Gazzola and Keysers 2009) showed that additional cortical areas have been found to be active during action observation and execution: the dorsal premotor cortex, the superior parietal lobule, the primary and secondary somatosensory cortices and the middle temporal cortex. These additional activations could enrich and complement the information available about the observed actions of others provided by the "standard" parieto-premotor mirror network (Rizzolatti and Sinigaglia 2010). Our data provide the first demonstration of such a wider network of cortical areas during action observation in children.

Finally, to our knowledge, this study represents the first empirical evidence on the investigation of the parietopremotor mirror network in which a children template of MNI is used. The introduction of a pediatric MNI template in the localization of brain sources allowed to approach the solution of the "inverse problem" with greater rigor and accuracy (for a discussion on EEG Source Imaging see
Michel et al. 2004). Such methodological aspect contributes to better address the issue of source localization analysis in the study of the MM in children.

In summary, this study provides the first description of two different parieto-premotor circuits activated by the observation of object-related hand reaching-to-grasping motor acts in children. The activation of these circuits is modulated in time, and influenced by the amount of visual information available about the hand grasping-related portion of the observed motor acts.

Acknowledgments This research was supported by a fellowship by Fondazione Monte Parma to C. B., by the EU grant Marie-Curie Initial Training Network, "TESIS: Towards an Embodied Science of Inter Subjectivity" (FP7-PEOPLE-2010-ITN, 264828) to V.G., and by the "Ricerca Finalizzata 2007-Programma Strategico Inquiry into disruption of intersubjective equipment in autistic spectrum disorders in childhood". C.B., T.R. and C.M. were supported by the National Center of Competence in Research (NCCR) "SYNAPSYThe Synaptic Bases of Mental Diseases" financed by the Swiss National Science Foundation (Grant No. 51AU40_125759). The Cartool software (http://brainmapping.unige.ch/Cartool.htm) has been programmed by Denis Brunet and is supported by the Center for Biomedical Imaging of Geneva and Lausanne, Switzerland.

\section{References}

Bernier R, Dawson G, Webb S, Murias M (2007) EEG mu rhythm and imitation impairments in individuals with autism spectrum disorder. Brain Cogn 64(3):228-237

Boria S, Fabbri-Destro M, Cattaneo L, Sparaci L, Sinigaglia C, Santelli E, Cossu G, Rizzolatti G (2009) Intention understanding in autism. PLoS One 4(5):e5596

Brass M, Schmitt RM, Spengler S, Gergely G (2007) Investigating action understanding: inferential processes versus action simulation. Curr Biol 17:2117-2121

Brett M (2006) The MNI brain and the Talairach atlas. http://imaging. mrc-cbu.cam.ac.uk/imaging/MniTalairach. Accessed 12 Dec 2012

Brunet D, Murray MM, Michel CM (2011) Spatiotemporal analysis of multichannel EEG: CARTOOL. Comput Intell Neurosci 2011:813870. doi:10.1155/2011/813870

Cattaneo L, Fabbi-Destro M, Boria S, Pieraccini C, Monti A, Cossu G, Rizzolatti G (2007) Impairment of actions chains in autism and its possible role in intention understanding. Proc Natl Acad Sci USA 104:17825-17830

Culham JC, Valyear KF (2006) Human parietal cortex in action. Curr Opin Neurobiol 16(2):205-212

Dapretto M, Davies MS, Pfeifer JH, Scott AA, Sigman M, Bookheimer SY, Iacoboni M (2006) Understanding emotions in others: mirror neuron dysfunction in children with autism spectrum disorders. Nat Neurosci 9(1):28-30

de Peralta G, Menendez R, Gonzalez Andino S, Lantz G, Michel CM, Landis T (2001) Noninvasive localization of electromagnetic epileptic activity. I. Method descriptions and simulations. Brain Topogr 14(2):131-137

di Pellegrino G, Fadiga L, Fogassi L, Gallese V, Rizzolatti G (1992) Understanding motor events: a neurophysiological study. Exp Brain Res 91(1):176-180

Fabbri-Destro M, Cattaneo L, Boria S, Rizzolatti G (2009) Planning actions in autism. Exp Brain Res 192(3):521-525. doi:10.1007/ s00221-008-1578-3 
Ferrari PF, Vanderwert RE, Paukner A, Bower S, Suomi SJ, Fox NA (2012) Distinct EEG amplitude suppression to facial gestures as evidence for a mirror mechanism in newborn monkeys. J Cogn Neurosci 24(5):1165-1172. doi:10.1162/jocn_a_00198 Epub 2012 Jan 30

Filimon F, Nelson JD, Hagler DJ, Sereno MI (2007) Human cortical representations for reaching: mirror neurons for execution, observation, and imagery. Neuroimage 37(4):1315-1328

Fonov VS, Evans AC, Botteron K, Almli CR, McKinstry RC, Collins DL, BDCG (2011) Unbiased average age-appropriate atlases for pediatric studies. NeuroImage 54(1):313-327. doi:10.1016/j. neuroimage.2010.07.033

Fonov VS, Evans AC, McKinstry RC, Almli CR, Collins DL (2009) Unbiased nonlinear average age-appropriate brain templates from birth to adulthood. NeuroImage vol 47 (supplement 1). Organization for Human Brain Mapping 2009 Annual Meeting. doi: 10.1016/S1053-8119(09)70884-5

Gallese V, Sinigaglia C (2011) What is so special about embodied simulation? Trends Cogn Sci. 15(11):512-519

Gallese V, Fadiga L, Fogassi L, Rizzolatti G (1996) Action recognition in the premotor cortex. Brain 119:593-609

Gallese V, Rochat M, Cossu G, Sinigaglia C (2009) Motor cognition and its role in the phylogeny and ontogeny of action understanding. Dev Psychol. 45(1):103-113

Gallese V, Rochat MJ, Berchio C (2012) The mirror mechanism and its potential role in autism spectrum disorder. Dev Med Child Neurol 55(1):15-22. doi:10.1111/j.1469-8749.2012.04398.x

Gazzola V, Keysers C (2009) The observation and execution of actions share motor and somatosensory voxels in all tested subjects: single-subject analyses of unsmoothed fMRI data. Cereb Cortex 19(6):1239-1255. doi:10.1093/cercor/bhn181

Iacoboni M, Molnar-Szakacs I, Gallese V, Buccino G, Mazziotta JC, Rizzolatti G (2005) Grasping the intentions of others with one's own mirror neuron system. PLoS Biol 3(3):e79

Kohler E, Keysers C, Umiltà MA, Fogassi L, Gallese V, Rizzolatti G (2002) Hearing sounds, understanding actions: action representation in mirror neurons. Science 297(5582):846-848

Lepage JF, Théoret H (2006) EEG evidence for the presence of an action observation-execution matching system in children. Eur $\mathbf{J}$ Neurosci 23(9):2505-2510

Lepage JF, Théoret H (2007) The mirror neuron system: grasping others' actions from birth? Dev Sci. 10(5):513-523

Marshall PJ, Meltzoff AN (2011) Neural mirroring systems: exploring the EEG mu rhythm in human infancy. Dev Cogn Neurosci 1(2):110-123. doi:10.1016/j.den.2010.09.001

Michel CM, Murray MM (2012) Towards the utilization of EEG as a brain imaging tool. Neuroimage. 61(2):371-385. doi:10.1016/j. neuroimage.2011.12.039

Michel CM, Murray MM, Lantz G, Gonzalez S, Spinelli L, Grave de Peralta R (2004) EEG source imaging. Clin Neurophysiol 115(10):2195-2222

Molenberghs P, Hayward L, Mattingley JB, Cunnington R (2012) Activation patterns during action observation are modulated by context in mirror system areas. Neuroimage 59(1):608-615

Murray MM, Brunet D, Michel CM (2008) Topographic ERP analyses: a step-by-step tutorial review. Brain Topogr 20(4):249-264. doi:10.1007/s10548-008-0054-5

Muthukumaraswamy SD, Johnson BW (2004) Changes in rolandic mu rhythm during observation of a precision grip. Psychophysiology 41(1):152-156
Muthukumaraswamy SD, Johnson BW, McNair NA (2004) Mu rhythm modulation during observation of an object-directed grasp. Brain Res Cogn Brain Res 19(2):195-201

Nyström P (2008) The infant mirror neuron system studied with high density EEG. Soc Neurosci 3(3-4):334-347. doi:10.1080/ 17470910701563665

Nyström P, Ljunghammar T, Rosander K, von Hofsten C (2011) Using mu rhythm desynchronization to measure mirror neuron activity in infants. Dev Sci. 14(2):327-335

Oberman LM, Hubbard EM, McCleery JP, Altschuler EL, Ramachandran VS, Pineda JA (2005) EEG evidence for mirror neuron dysfunction in autism spectrum disorders. Brain Res Cogn Brain Res 24(2):190-198

Oberman LM, Ramachandran VS, Pineda JA (2008) Modulation of mu suppression in children with autism spectrum disorders in response to familiar or unfamiliar stimuli: the mirror neuron hypothesis. Neuropsychologia. 46(5):1558-1565

Oberman LM, McCleery JP, Hubbard EM, Bernier R, Wiersema JR, Raymaekers R, Pineda JA (2012) Developmental changes in mu suppression to observed and executed actions in autism spectrum disorders. Soc Cogn Affect Neurosci 8(3):300-304

Ortigue S, Sinigaglia C, Rizzolatti G, Grafton ST (2010) Understanding actions of others: the electrodynamics of the left and right hemispheres. A high-density EEG neuroimaging study. PLoS One 5(8):e12160. doi:10.1371/journal.pone.0012160

Pineda JA (2005) The functional significance of mu rhythms: translating "seeing" and "hearing" into "doing". Brain Res Brain Res Rev 50(1):57-68

Reid VM, Striano T, Iacoboni M (2011) Neural correlates of dyadic interaction during infancy. Dev Cogn Neurosci. 1(2):124-130. doi:10.1016/j.den.2011.01.001

Rizzolatti G, Sinigaglia C (2010) The functional role of the parietofrontal mirror circuit: interpretations and misinterpretations. Nat Rev Neurosci 11(4):264-274

Rizzolatti G, Fadiga L, Gallese V, Fogassi L (1996) Premotor cortex and the recognition of motor actions. Brain Res Cogn Brain Res 3(2):131-141

Southgate V, Csibra G (2009) Inferring the outcome of an ongoing novel action at 13 months. Dev Psychol 45(6):1794-1798. doi:10.1037/a0017197

Spinelli L, Andino SG, Lantz G, Seeck M, Michel CM (2000) Electromagnetic inverse solutions in anatomically constrained spherical head models. Brain Topogr 13(2):115-125

Streltsova A, Berchio C, Gallese V, Umilta' MA (2010) Time course and specificity of sensory-motor alpha modulation during the observation of hand motor acts and gestures: a high density EEG study. Exp Brain Res 205(3):363-373. doi:10.1007/s00221-0102371-7

Umiltà MA, Kohler E, Gallese V, Fogassi L, Fadiga L, Keysers C, Rizzolatti G (2001) I know what you are doing. A neurophysiological study. Neuron 31(1):155-165

van Elk M, van Schie HT, Hunnius S, Vesper C, Bekkering H (2008) You'll never crawl alone: neurophysiological evidence for experience-dependent motor resonance in infancy. Neuroimage 43(4):808-814. doi:10.1016/j.neuroimage.2008.07.05 